Niklas Briest*, Heyno Garbe und Stefan Potthast

\title{
Bewertung der Formveränderung transienter Signale in GTEM-Zellen
}

\author{
Evaluation of the deformation of transient signals within GTEM cells
}

DOI 10.1515/teme-2016-0076

Eingang 1. Dezember 2016; überarbeitet 27. Dezember 2016; angenommen 29. Dezember 2016

Zusammenfassung: Die Verifikation von transversal elektromagnetischen (TEM) Wellenleitern, speziell GTEMZellen, beruht auf der Norm IEC 61000-4-20 im Frequenzund Zeitbereich. Die Verifikation im Zeitbereich basiert auf einem Nuklear elektromagnetischen Puls (NEMP) mit einer doppeltexponentiellen Form, der über die Parameter der Anstiegszeit und Pulsbreite in festgelegten Parametergrenzen definiert ist. Eine Verifikation der GTEM-Zelle für andere transiente Signalformen ist anhand dieser Parameter nicht zu realisieren. Des Weiteren lassen sich mit diesem Ultra-Breitbandpuls (UWB) keine eventuell kritischen Frequenzen des betrachteten Wellenleiters identifizieren. Im Hinblick auf neue Signalformen in der Nachrichtentechnik und transiente Signale die Anwendung in der elektromagnetischen Verträglichkeit (EMV) finden, ist eine Verifikation von TEM-Wellenleiter für diese Signalformen zwingend notwendig. Die in diesem Beitrag vorgestellte Methode ermöglicht die Qualifizierung eines TEMWellenleiters für jedes beliebige transiente Signal. Unter Anwendung der Korrelation wird ein Signalvergleich durchgeführt, der ein Maß für die Übertragungsqualität der betrachteten Wellenleiters für die jeweilige Signalform darstellt.

Schlüsselwörter: TEM-Wellenleiterverifikation, Störfestigkeitstest in der EMV.

The results shown in this paper were partly produced with the support of the Bundeswehr Research Institute for Protective Technologies, NBC-Protection in Munster, Germany. Contract Number E/E590/CF148.

*Korrespondenzautor: Niklas Briest, Institut für Grundlagen der Elektrotechnik und Messtechnik, Leibniz Universität Hannover, E-Mail: briest@geml.uni-hannover.de.

http://orcid.org/0000-0003-2352-7975

Heyno Garbe: Institut für Grundlagen der Elektrotechnik und Messtechnik, Leibniz Universität Hannover

Stefan Potthast: Wehrwissenschaftliches Institut für

Schutztechnologien - ABC-Schutz, Munster

\begin{abstract}
The verification of transversal electromagnetic (TEM) waveguides, especially GTEM cells, is described in the standard IEC 61000-4-20 in the frequency and time domain. The verification in time domain is based on a nuclear electromagnetic pulse (NEMP) with a double exponential waveform, with is defined by two parameters, the rise time and pulsewidth, and the given parameter limits. A verification of the transmission qualitiy of a GTEM cell for arbitrary transient signals based on these parameters is not possible. Furthermore, it is not possible to identify critical frequencies of the performed waveguide with such an ultra-wideband pulse. Due to new upcoming signal shapes in the communications engineering and the use of transient signals in electromagnetic compatibility (EMC) issues, a verification of waveguides for these waveform is essential. The method presented in this paper allows the qualification of a TEM waveguide for arbitrary transient signals. A signal comparison based on a correlation is performed, which quantifies the transmission quality of a waveguide for the performed transient shape.
\end{abstract}

Keywords: TEM waveguide verification, EMC immunity testing.

\section{Einleitung}

Transversal elektromagnetische (TEM) Wellenleiter dienen vorwiegend Störaussendungs- und Störfestigkeitsuntersuchungen [6] von elektrischen und elektronischen Geräten und stellen eine gängige Alternative zum Freifeld Messplatz dar. Im Speziellen eignen sich GTEM-Zellen da$\mathrm{zu}$ einen großen Frequenzbereich bei den Untersuchungen abzudecken. Eine GTEM-Zelle lässt sich als theoretisch perfekt abgeschlossenes, sich im Querschnitt mit der Länge aufweitendes Koaxialkabel beschreiben (Abbildung 1). Sie besteht aus einem Innenleiter - hier als Septum bezeichnet - und einer metallischen Hülle, die als Außenleiter fungiert. Der Abschluss wird über ein $50 \Omega$ Widerstandsnetzwerk realisiert. Die Wellenausbreitung findet theoretisch im TEM-Mode statt, wonach das $E$-Feld - $y$-Richtung des Wellenleiterkoordinatensystems 
- senkrecht zum $H$-Feld - $x$-Richtung - steht (siehe Abbildung 2) [2]. Der Poynting-Vektor berechnet sich aus dem Kreuzprodukt der beiden Felder und beschreibt die Leistungsdichte der Welle. Eine Reflektion der sich ausbreitenden Welle wird über die an der Rückwand angebrachten Schaumabsorber reduziert. Der Einsatz von TEM-Wellenleitern bietet zwei wesentliche Vorteile gegenüber einem Freifeld. Die wesentlich geringere Baugröße ermöglicht eine Verwendung des Wellenleiters innerhalb eines Labors. Des Weiteren verhindert der als metallische Hülle realisierte Außenleiter jegliche Beeinflussung des Messaufbaus durch elektromagnetische Einflüsse aus der Umwelt. Daraus resultiert auch die Möglichkeit den Prüfling mit hohen Feldstärken im kV-Bereich zu beaufschlagen, ohne sich einer Gefährdung durch elektromagnetische Wellen auszusetzen.

Die Validierung von GTEM-Zellen ist in der IEC Norm 61000-4-20 festgelegt. Dabei wird zwischen der Validierung im Frequenz- und Zeitbereich unterschieden. Im Frequenzbereich beschreibt die Normung zwei zu untersuchende Kriterien - die Gleichförmigkeit des E-Feldes und

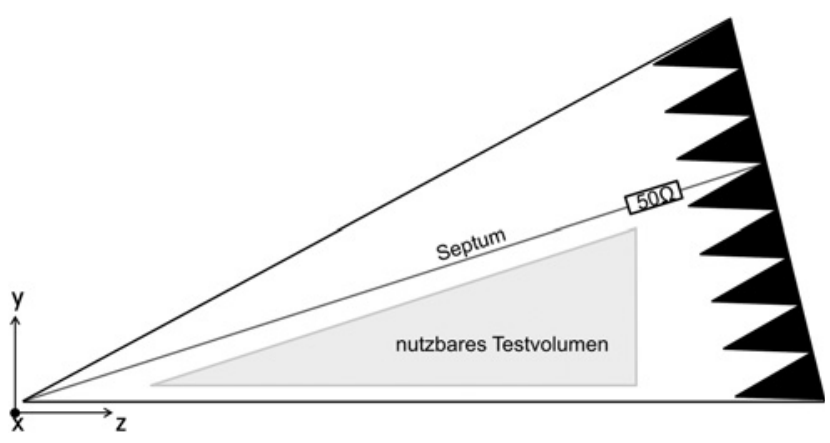

Abbildung 1: Schematischer Längsschnitt einer GTEM-Zelle.

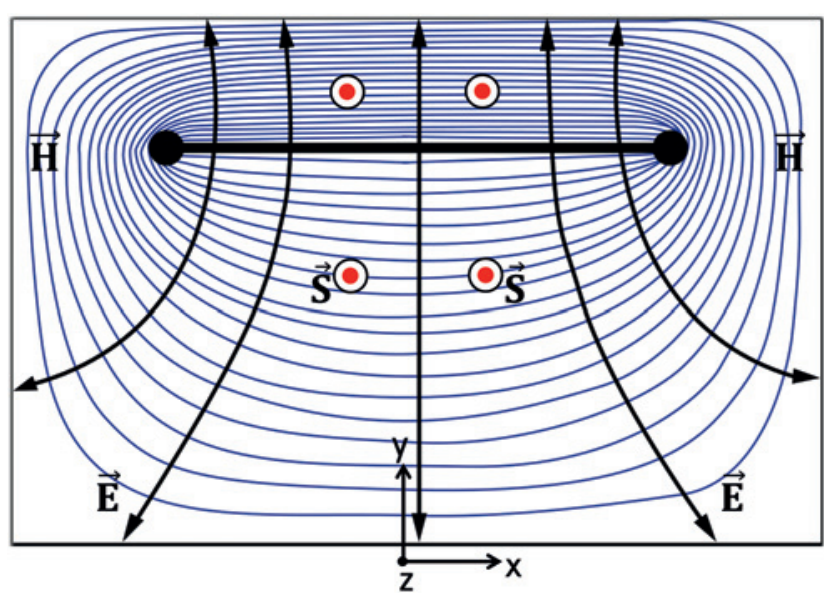

Abbildung 2: TEM-Mode in GTEM-Zelle. die Ausbreitung der elektromagnetischen Welle im TEMMode. Die IEC 61000-4-20 Annex C [1] umfasst die Validierung einer GTEM-Zelle im Zeitbereich. Darin wird ein doppeltexponentieller Puls mit einer Anstiegszeit $\left(t_{\text {rise }}\right)$ und einer Pulsbreite $\left(t_{\text {fwhm }}\right)$ definiert. Der Puls wird mit dem folgenden mathematischen Ausdruck beschrieben

$$
E(t)=E_{\text {peak }} \cdot k\left(e^{-\beta t}-e^{-\alpha t}\right) \text { in } \frac{\mathrm{V}}{\mathrm{m}} .
$$

Die Validierung im Zeitbereich schreibt vor, dass die in der GTEM-Zelle gemessene $y$-Komponente des $E$-Feldes und dessen definierende Parameter nur wie folgt abweichen dürfen:

$-\quad t_{\text {rise }}=2,25 \mathrm{~ns} \pm 0,25 \mathrm{~ns}$

- $t_{\mathrm{fwhm}}=27,5 \mathrm{~ns} \pm 2,5 \mathrm{~ns}$

Diese Parameter, Anstiegszeit und Pulsbreite, sind jedoch nicht auf jedes beliebige Signal übertragbar und die Übertragungsqualität des Wellenleiters, ist unter Anwendung des Annex C, nicht für jedes beliebige transiente Signal erfassbar. Des Weiteren besteht die Frage, inwiefern der im Annex C beschriebene UWB-Puls stellvertretend für transiente Signale eingesetzt werden und speziell einzelne kritische Frequenzen aufdecken kann. Es besteht der Anspruch eine Methode zu entwickeln, die es ermöglicht GTEM-Zellen und deren Übertragungscharakteristik für jede beliebige Signalform zu qualifizieren. Eine Methode, die diesen Ansatz verfolgt, wird innerhalb dieses Beitrages vorgestellt.

\section{Verifikationsmethode}

Da sich die Norm für GTEM-Zellen, nach aktuellem Stand, im Zeitbereich auf eine Signalform beschränkt und Signalparameter als qualifizierende Kriterien angeführt werden, die nicht auf alle beliebigen Signale übertragbar sind, soll die hier vorgestellte Methode auf beliebige transiente Signale übertragbar sein. Die prinzipielle Idee beruht auf dem Vergleich zweier Signale. Über diesen Vergleich soll eine Beurteilung der Übertragungsqualität erfolgen. In vorangehenden Arbeiten von [4] und [3] wird der Korrelationskoeffizient nach Pearson für einen Vergleich zweier Signale herangezogen, mittels dessen eine Aussage über die Ähnlichkeit der Signale getroffen werden kann. Der Pearson-Korrelationskoeffizient ist wie in (2) angeführt definiert:

$$
\rho(X, Y)=\frac{\operatorname{Cov}(X, Y)}{\delta(X) \delta(Y)} .
$$

Der Koeffizient berechnet sich aus der Kovarianz zwischen zwei Signalen, welche ein Maß für den monotonen $\mathrm{Zu}$ sammenhang der beiden Signale darstellt. Die Kovarianz 


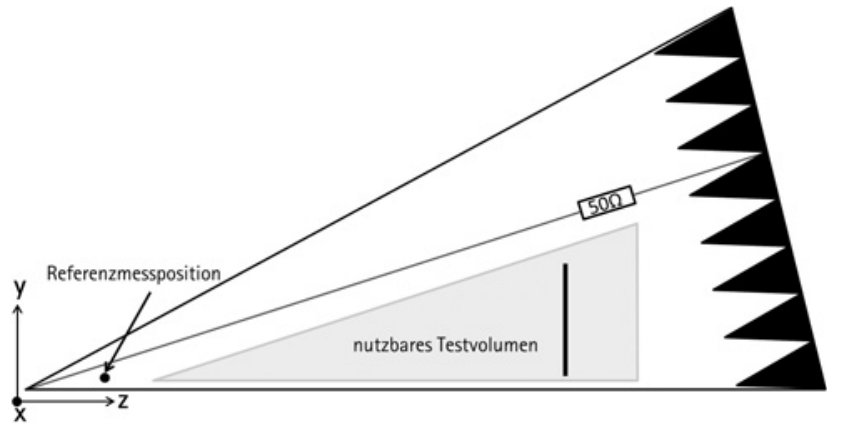

Abbildung 3: TEM-Mode in GTEM-Zelle.

bildet jedoch nur eine Tendenz bzw. eine Richtung des Zusammenhangs ab. Eine Normierung auf die Standardabweichung der beiden Signale erfolgt anschließend. Für den Vergleich wird die Hauptkomponente des elektrischen Feldes $E_{y}$ im Zeitbereich herangezogen.

Um eine Aussage über die Übertragungscharakteristik treffen zu können, muss der Vergleich zwischen einem unverfälschten Signal und den zu testenden Signalen im Prüfvolumen der GTEM-Zelle erfolgen. Nach [5] breiten sich elektromagnetische Wellen bei kleinen Zellenquerschnitten unter TEM-Bedingungen aus. Eine Anregung höherer Moden, durch die eine nicht formtreue Übertragung verursacht werden kann, findet hier nicht statt. Daher ist an kleinen Zellenquerschnitten innerhalb der GTEM-Zelle eine formtreue Wandlung des Spannungssignals in ein Feldsignal anzunehmen. Das Prüfvolumen wird - angelehnt an die Validierung eines TEM-Wellenleiters im Frequenzbereich - entlang einer $x$ - $y$-Ebene bei einer konstanten $z$-Position vermessen. Dabei wird die Ebene in $n$ verschiedene Punkte gerastert. Die beschriebenen Messpositionen sind in Abbildung 3 dargestellt.
Der Korrelationskoeffizient ergibt sich nach (3), wobei $E_{y \text {,ref }}$ das gemessene $E$-Feld in $y$-Richtung am eingezeichneten Referenzpunkt und $E_{y, \text { ts }}$ das im Prüfvolumen beschreibt;

$$
\rho\left(E_{y, \text { ref }}, E_{y, \mathrm{ts}}\right)=\frac{\frac{1}{N} \sum_{k=1}^{N} E_{y, \text { ref }} \cdot E_{y, \mathrm{ts}}}{\sqrt{\frac{1}{N} \sum_{k=1}^{N} E_{y, \text { ref }}^{2}} \cdot \sqrt{\frac{1}{N} \sum_{k=1}^{N} E_{y, \mathrm{ts}}^{2}}}
$$

mit

$$
\rho\left(E_{y, \text { ref }}\right)=E_{y, \text { ref }}[k]-\bar{E}_{y, \text { ref }}
$$

und

$$
\rho\left(E_{y, \mathrm{ts}}\right)=E_{y, \mathrm{ts}}[k]-\bar{E}_{y, \mathrm{ts}} .
$$

Bei der Vermessung der Feldsignale wird, sofern möglich, auf baugleiche Sensoren zurückgegriffen. Dies vereinfacht die Auswertung in der Anwendung der Korrelation. Sofern keine baugleichen Sensoren zu Verfügung stehen, muss der Antennenfaktor des jeweiligen Sensors herausgerechnet werden. Die Feldstärkendifferenz zwischen den zwei Messpositionen muss jedoch nicht berücksichtigt werden, da diese den Koeffizienten nicht beeinflusst. Eine wesentliche Fragestellung liegt in der korrekten Länge $\left(t_{\text {Signallänge }}\right)$ der zu korrelierenden Signale und den dafür maßgeblichen Start- und Endpunkt des Signals. Zwei Aspekte sollen bei dieser Signalfensterung berücksichtigt werden. Das transiente Signal soll entlang der $x$ - $y$-Ebene formtreu gegenüber dem Ausgangssignal abgebildet werden und die ursprünglichen Signalinformationen beinhalten. Das hierfür festgelegte Signalfenster wird mit $t_{1}$ beschrieben. Des Weiteren sollen eventuelle verzerrende Einflüsse der GTEM-Zelle in dem Korrelationskoeffizienten,

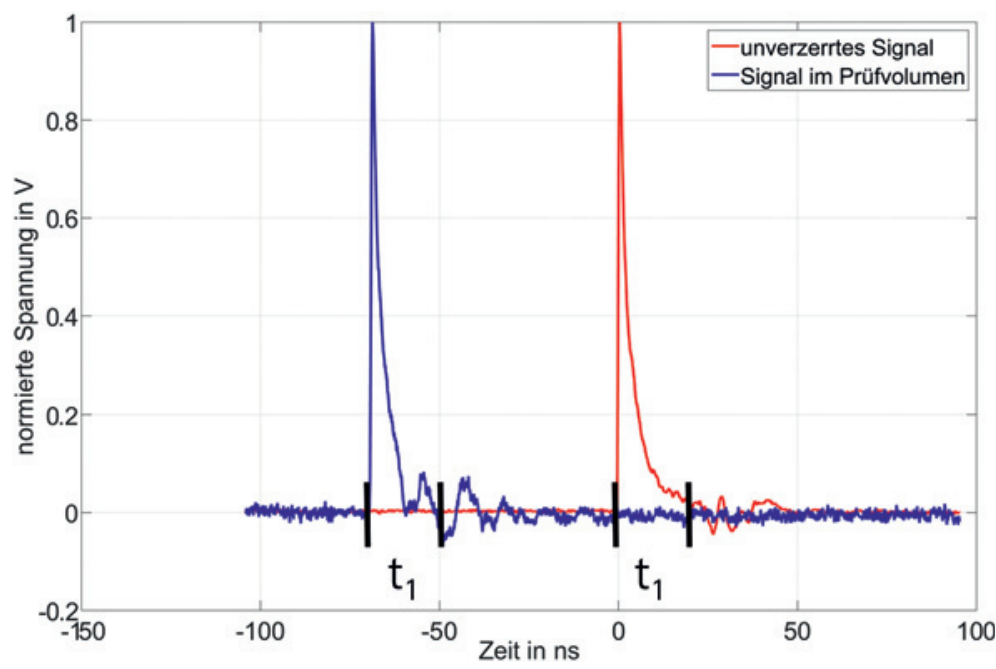

Abbildung 4: Signalfenster nach $t_{1}$. 


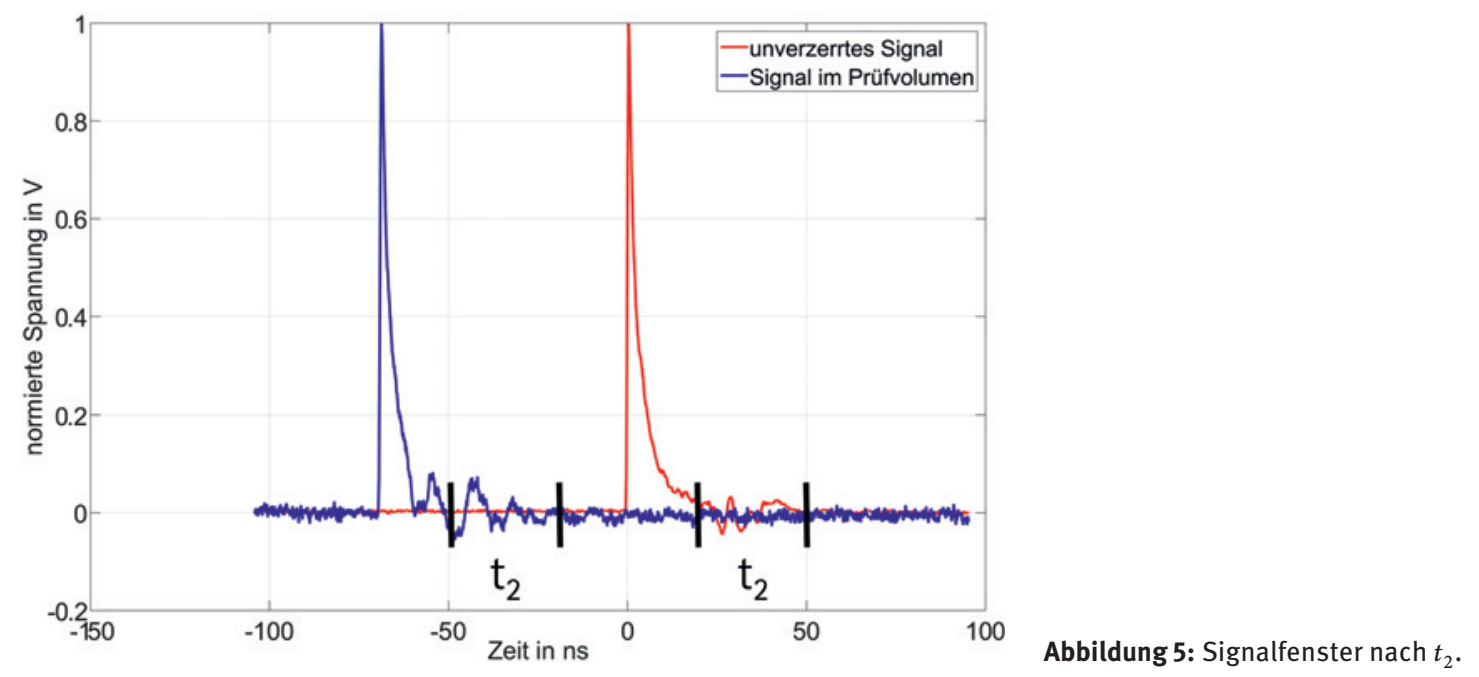

mit der Erweiterung des Signalfensters um $t_{2}$, berücksichtigt werden.

$$
t_{\text {Signallänge }}=t_{1}+t_{2} \text {. }
$$

Abhängig vom Signal-Rausch-Verhältnis (SNR) kann der Start- $\left(t_{\text {Start }}\right)$ und Endwert $\left(t_{\text {Ende }}\right)$ für das Referenzsignal, gemäß Abbildung 4, zu

$$
t_{1}=t_{\text {Ende }}-t_{\text {Start }}
$$

gewählt werden.

Über die Laufzeit zwischen der Referenzposition und der Messposition auf der Gleichförmigkeitsebene wird unter Annahme der Lichtgeschwindigkeit $c_{0}$ der Startpunkt der dort gemessenen Signale festgelegt. Um eventuelle Eigenschaften der GTEM-Zelle auf die Signale berücksichtigen zu können, muss der Endpunkt der Fensterung in Abhängigkeit zu der Zellengeometrie gewählt werden. Mögliche Reflektionen an der Zellenrückwand können berücksichtigt werden, in dem der Endpunkt der Fensterung um die zweifache Laufzeit von der Messposition zu den Absorbern $d_{\text {Absorber }}$ (siehe Abbildung 5), unter erneuter Annahme der Ausbreitungsgeschwindigkeit $c_{0}$, über den eigentlichen Signalendpunkt hinaus verlängert wird.

$$
t_{2}=\frac{2 \cdot d_{\text {Absorber }}}{c_{0}}
$$

Die sich daraus, für die im Prüfvolumen gemessenen Signale, ergebende Signallänge $t_{\text {Signallänge }}$ wird auf das Referenzsignal projiziert.

Die nach diesen Vorgaben erfolgte Fensterung ermöglicht die Reduzierung der Signalinformation auf die ursprüngliche Signalform und berücksichtigt Einflüsse der betrachteten GTEM-Zelle. Der anschließende Vergleich der
Signale im Prüfvolumen über die Korrelation beschreibt abschließend die Übertragungsqualität der GTEM-Zelle für das betrachtete transiente Signal und identifiziert Abweichungen vom Referenzsignal.

\section{Anwendung der Methode für die GTEM1250}

Die beschriebene Verifikationsmethode wird auf die GTEM1250 von EMCO angewendet. Die Septumshöhe am Ende des nutzbaren Prüfvolumens beträgt $1250 \mathrm{~mm}$. Die für die Messung genutzte Gleichförmigkeitsebene ist in Abbildung 6 dargestellt und bei einer Septumshöhe von $1000 \mathrm{~mm}$ platziert. Sie umfasst eine Fläche von $1 \mathrm{~m}^{2}$ und wird in neun Messpunkte unterteilt.

Die Übertragungscharakteristik soll für einen doppeltexponentiellen Puls untersucht werden um einen

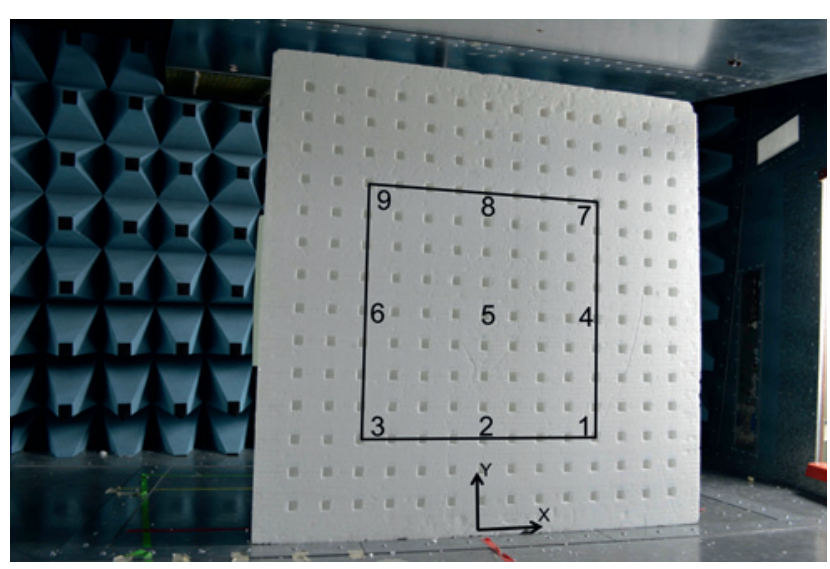

Abbildung 6: Gleichförmigkeitsebene bei $1000 \mathrm{~mm}$ Septumshöhe. 
direkt Zusammenhang zur Verifikationsmethode der IEC 61000-4-20 herzustellen. Des Weiteren soll anhand einer anderen Signalform überprüft werden, inwieweit die vorgestellte Methode Verzerrungen beliebiger Signalformen bewerten kann und sich damit die Übertragungscharakteristik eines TEM-Wellenleiters für dieses Signal beschreiben lässt. Die hier vorgestellte Methode besitzt in dem Korrelationskoeffizienten nach Pearson einen nicht-signalspezifischen Parameter als beurteilendes Kriterium, womit sich die Formtreue einer Wellenleiters für eine beliebige Signalform auswerten lässt.

Eine Signalform die in der Nachrichtentechnik und in der EMV eine zunehmende Rolle spielt sind gedämpft schwingende Pulse (DS), womit eine formtreue Übertragung in GTEM-Zellen im Hinblick auf Störfestigkeitstests unabdingbar sind. Ein großer Vorteil dieser Pulse bzw. der Validierung von GTEM-Zellen mit diesen Pulsformen liegt in dem vergleichsweise schmalen Frequenzspektrum, was erneut den Bedarf an dieser Methode unterstreicht. Denn die Mittenfrequenz eines solchen DS lässt sich auf eventuell kritische Frequenzen einer GTEM-Zelle abstimmen und damit die Übertragungsqualität von transienten Signalen mit wesentlichen Spektralanteilen bei speziellen Frequenzen beurteilen.

\subsection{Verifizierung der GTEM1250 für einen UWB-Puls}

Die GTEM1250 soll für einen UWB-Puls mit einer ebenfalls doppeltexponentiellen Form verifiziert werden. Dieser wird mit dem PBG3 von Kenntech Instruments erzeugt und wird über die Anstiegszeit $t_{\text {rise }}$ und die Pulsbreite $t_{\text {fwhm }}$ beschrieben. Dabei ist $t_{\text {rise }}$ mit 100 ps und $t_{\text {fwhm }}$ mit 3 ns angegeben. Das Verhältnis von Pulsbreite $\mathrm{zu}$ Anstiegszeit dieses Pulses ist im Vergleich zum in der IEC 61000-4-20 definierten Puls $\left(t_{\text {fwhm }} / t_{\text {rise }}=10\right)$ um das
Dreifache größer $\left(t_{\text {fwhm }} / t_{\text {rise }}=33\right)$. Die Pulsform ist im Zeitbereich über die folgende mathematische Form definiert

$$
U(t)=U_{0} \cdot\left(e^{-\beta t}-e^{-\alpha t}\right) .
$$

Dabei beschreibt der Parameter $\beta$ den Kehrwert der Rückenzeit $t_{\text {fall }}$ und $\alpha$ den Kehrwert der Anstiegszeit $t_{\text {rise }}$.

Die Erfassung des doppeltexponentiellen Pulses erfolgt über optoelektronische Sonden, EFS-105 der Firma enprobe. Diese können für Signale im Frequenzbereich zwischen $500 \mathrm{kHz}$ bis $3 \mathrm{GHz}$ eingesetzt werden. Die Abbildung 7 zeigt den Einsatz der optoelektronischen Sonden.

Die Positionierung an der Referenzposition und in der Gleichförmigkeitsebene innerhalb des Prüfvolumens der GTEM-Zelle erfolgt über Halterungen aus Polylactide (PLA), dessen elektromagnetischer Einfluss vernachlässigbar ist. Unter Einsatz der optoelektronischen Sonden wird die $y$-Komponente des $E$-Feldes gemessen. Aufgrund der parallelen Aufzeichnung ist die Unabhängigkeit von der Reproduzierbarkeit des Signalgenerators innerhalb dieser Methode gewahrt. Anschließend an die Vermessung der Gleichförmigkeitsebene werden die beschriebene Signalverarbeitung und die Berechnung des Korrelationskoeffizienten durchgeführt. Die Auswertung des Korrelationskoeffizienten wird in einer Heatmap umgesetzt. Dies ermöglicht eine schnelle und unkomplizierte Auswertung der Übertragungscharakteristik des TEM-Wellenleiters.

Aus Abbildung 8 ergeben sich die, für die neun Messpositionen berechneten, Korrelationskoeffizienten für den eingespeisten UWB-Puls.

Der berechnete Korrelationskoeffizient für die Übertragung des doppeltexponentiellen Pulses innerhalb der GTEM1250 liegt zwischen 0,976 und 0,986 und stellt damit eine sehr hohe Übertragungsqualität dar. Es ist eine Symmetrie zur $y$-Achse bei $x=0$ zu verzeichnen. Des Weiteren ist eine kontinuierliche Zunahme des Koeffizienten entlang der $y$-Achse zu erkennen. In Abbildung 9 ist der Vergleich zwischen dem am Referenzpunkt und
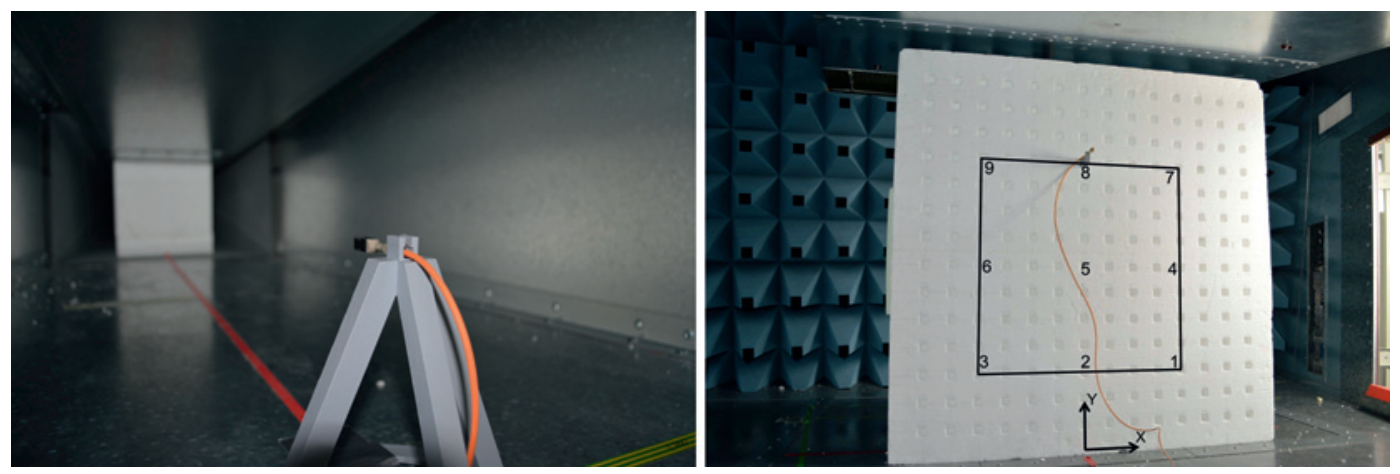

Abbildung 7: Einsatz der EFS-105 an Referenzposition und im Prüfvolumen. 


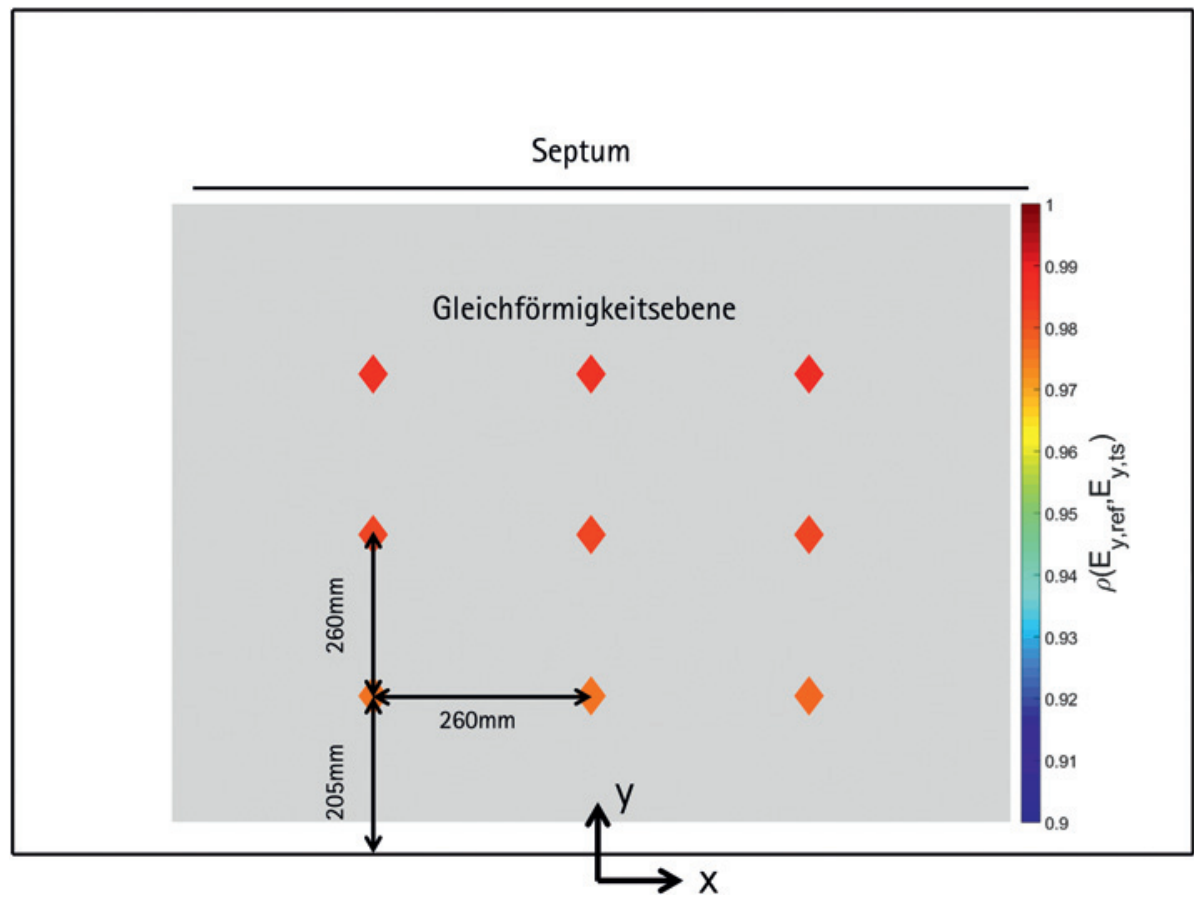

Abbildung 8: Korrelationskoeffizient für den UWB-Puls.
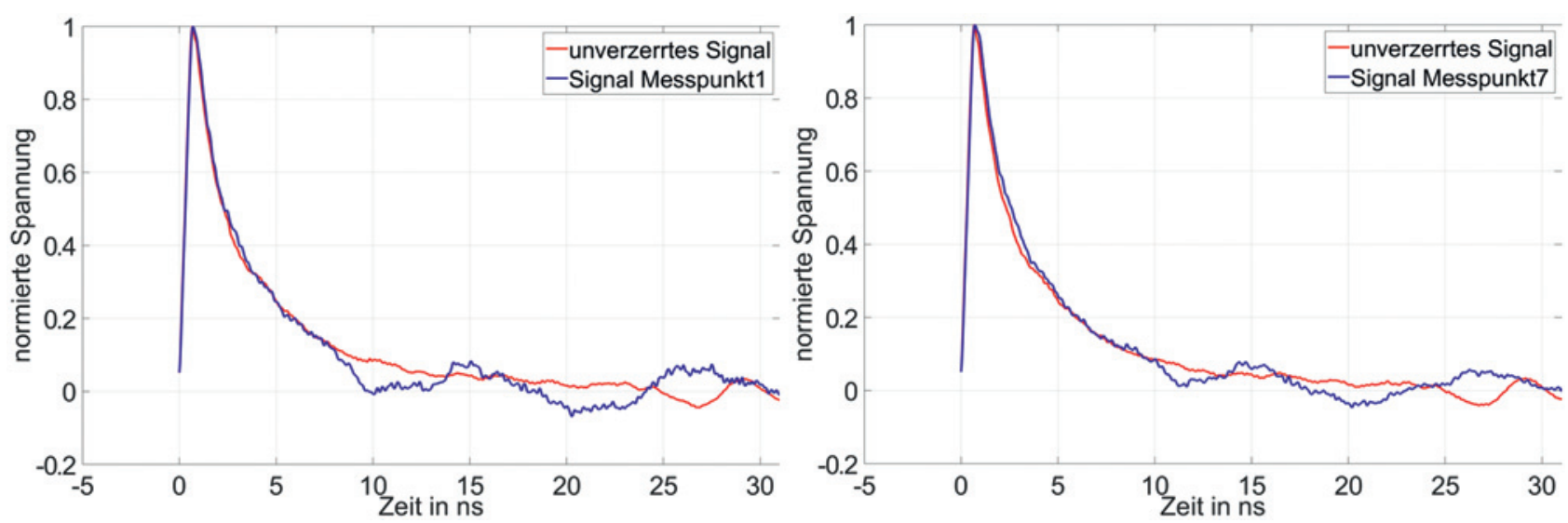

Abbildung 9: Einsatz der EFS-105 an Referenzposition und im Prüfvolumen.

zwei in der Gleichförmigkeitsebene gemessenen Signalen dargestellt. Unter Betrachtung der Signale im Zeitbereich lässt sich die getroffene Aussage über die hohe Übertragungsqualität der GTEM1250 für den gespeisten doppeltexponentiellen Puls stützen. Der Pulsanstieg und -rücken werden im Prüfvolumen nahezu formtreu abgebildet. Ein Vergleich der Anstiegszeit und Pulsbreite kann unter Anwendung der vorgestellten Methode entfallen, was die Beurteilung der Übertragungsqualität vereinfacht und beschleunigt.

\subsection{Verifizierung der GTEM1250 für einen DS-Puls}

Die Übertragungscharakteristik der GTEM1250 für den verwendeten UWB-Puls konnte unter Anwendung der vorgestellten Methode bewertet werden. Im weiteren Verlauf des Beitrages soll weiterhin diskutiert werden, inwiefern sich die Methode auch in Anwendung auf einen DS-Puls eignet. Da es sich bei einem DS-Puls um einen vergleichsweise schmalbandigen transienten Puls handelt, besteht die Möglichkeit die Mittenfrequenz des Pulses abzustimmen. Im Vorfeld einer Validierung der GTEM1250 für einen DSPuls muss daher eine Diskussion über auszuwählende Frequenzen geführt werden. 
Im Gegensatz zu TEM-Wellenleitern wie die CrawfordZelle, welche aufgrund ihrer Bauform eine ausgewiesene Cut-off-Frequenz besitzt, ist eine GTEM-Zelle in einem nahezu ununterbrochenen Frequenzbereich nutzbar. Dennoch wird zunächst eine Analyse über die Existenz des TEM-Modes durchgeführt, um eventuelle kritische Frequenzen der GTEM1250 aufzudecken. Der TEM-Mode wird nach der IEC Norm 61000-4-20 über das Verhältnis von den sekundären $E$-Feld Komponenten, $E_{x}$ und $E_{z}$, zu der primären $E$-Feld Komponente, $E_{y}$, in einem Frequenzbereich von $30 \mathrm{MHz}$ bis $1 \mathrm{GHz}$ ausgewertet. Dabei muss das Verhältnis kleiner sein als $-6 \mathrm{~dB}$.

Die Auswertung erfolgt in den bereits betrachteten 9 Messpositionen der Gleichförmigkeitsebene. Dazu wird unter Einsatz einer triaxialen Messsonde die jeweilige Messposition in $E_{x}, E_{y}$ und $E_{z}$ vermessen, wobei die primäre $E$-Feldkomponente auf konstante $10 \mathrm{~V} \mathrm{~m}^{-1}$ geregelt wird, um eine verwertbare sekundäre Feldkomponente zu garantieren.

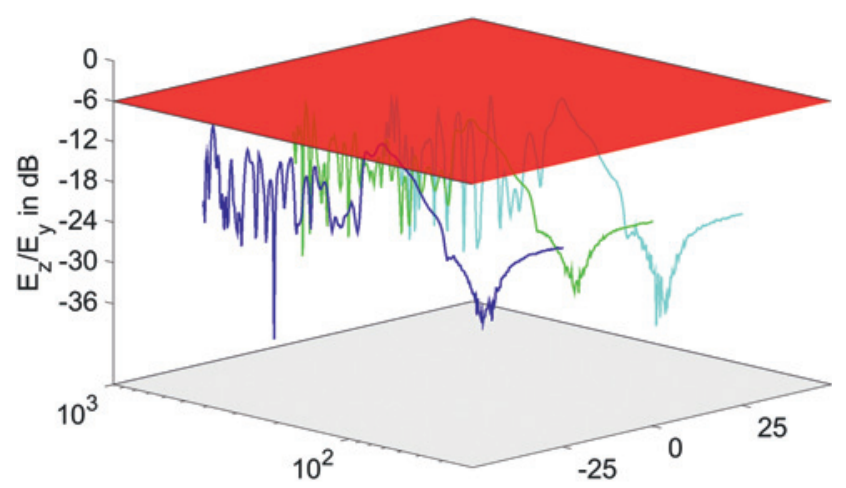

Frequenz in $\mathrm{MHz}$ $x$-Achse in $\mathrm{cm}$

Abbildung 10: Betrachtung des $\frac{E_{z}}{E_{y}}-$ Verhältnisses im Frequenzbereich.

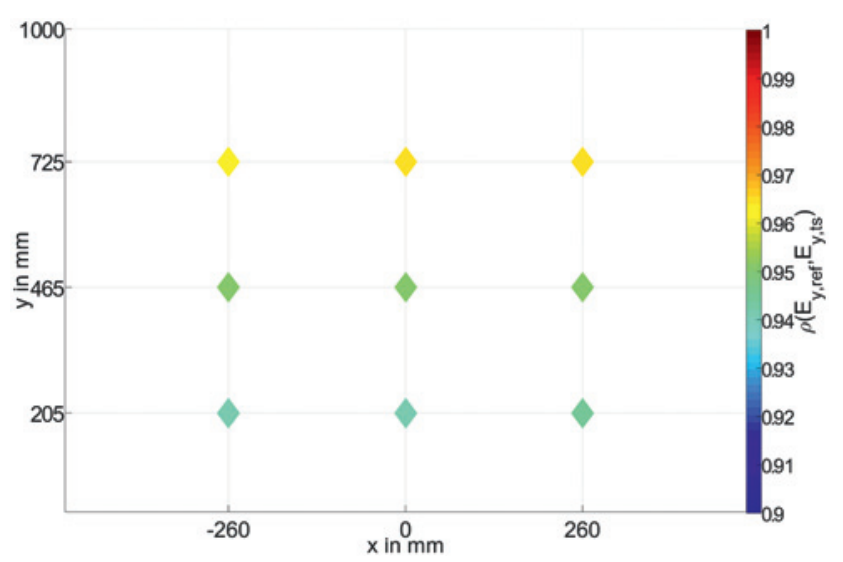

Abbildung 11: Korrelationskoeffizient für den $100 \mathrm{MHz}$ DS.
In der Abbildung 10 wird beispielhaft das Verhältnis von $E_{z}$ zu $E_{y}$ für die Messpunkte 4, 5 und 6 angeführt. Die $x$-Achse des Graphen umfasst die $x$-Koordinate der jeweiligen Messposition in der Gleichförmigkeitsebene, die $y$-Achse die Frequenz und die $z$-Achse das Verhältnis in $\mathrm{dB}$, wobei die $-6 \mathrm{~dB}$-Grenze als Fläche in der $x-y$-Ebene eingezeichnet ist.

Grundlegend lässt sich für die drei betrachteten Messpositionen über den gesamten Frequenzverlauf feststellen, dass das $-6 \mathrm{~dB}$-Kriterium eingehalten wird und nach der Definition der IEC 61000-4-20 der TEM-Mode verifiziert ist. Dennoch lassen sich starke Unterschiede und enorme Schwankungen über den Verlauf erkennen. So ist an den drei Messpositionen eine enorme Zunahme des Verhältnisses von $E_{z}$ zu $E_{y}$ bei einer Frequenz $100 \mathrm{MHz}$ zu verzeichnen. Eine Validierung der GTEM-Zelle für einen DS-Puls mit einer Mittenfrequenz $100 \mathrm{MHz}$ erscheint somit sinnvoll. Die Übertragungsqualität wird an den neun vorgestellten Messpositionen für die GTEM1250 ermittelt und ist in Abbildung 11 dargestellt.

Für den DS mit einer Mittenfrequenz von $100 \mathrm{MHz}$ wird ein Korrelationskoeffizient zwischen 0,941 und 0,965 ermittelt. Die Übertragungsqualität der GTEM1250 ist auch für diese Pulsform als gut zu beschreiben. Jedoch ist eine Abnahme gegenüber des für den eingespeisten UWB-Puls berechneten Korrelationskoeffizienten zu verzeichnen.

Das Ergebnis unterstreicht die Notwendigkeit der Erweiterung der von der IEC 61000-4-20 beschriebenen Validierung um die in diesem Beitrag vorgestellte Methode der TEM-Wellenleiter Validierung im Zeitbereich. Anhand des $100 \mathrm{MHz}$ DS Pulses lässt sich nachweisen, dass der UWB-Puls nicht jede transiente Pulsform, deren Spektrum innerhalb des Frequenzspektrums des UWB-Pulses liegt, repräsentiert. Daher ist es unabdingbar den eingesetzten TEM-Wellenleiter und dessen Übertragungscharakteristik für die Pulsform, die während Störfestigkeitsmessungen eingesetzt wird, zu verifizieren. Anhand der hier vorgestellten Pulsformen und der Beurteilung der Übertragungsqualität für diese Signale mit der hier beschriebenen Methode kann gezeigt werden, dass diese auf beliebige transiente Signale anwendbar ist.

\section{Zusammenfassung}

TEM-Wellenleiter stellen eine gängige Alternative zu Freifeld Messplätzen zur Durchführung von Störfestigkeitsund Störaussendungsmessungen dar. Die in der Norm IEC 61000-4-20 beschreibt die Durchführung dieser Messungen und umfasst die Validierung von TEMWellenleitern im Frequenz- und Zeitbereich. Der Annex C 
der Norm führt die Validierung im Zeitbereich aus. Diese basiert auf der Speisung eines doppeltexponentiellen Pulses. Diese Signalform wird über ihre Anstiegszeit und ihre Pulsbreite definiert. Auf Basis einer Auswertung dieser Parameter wird die Übertragungsqualität des Wellenleiters beurteilt. Eine Verifikation eines TEM-Wellenleiters für ein von der doppeltexponentiellen Form oder den darin definierten Parametergrenzen abweichendes Signal unter Anwendung des Annex C nicht umsetzbar. Im Hinblick auf das breitbandige Frequenzspektrum besteht die Frage inwiefern eine Speisung beliebiger Signal notwendig ist.

Inhalt dieses Beitrages ist die Entwicklung einer Methode zur Qualifizierung von TEM-Wellenleitern, insbesondere GTEM-Zellen, und deren Übertragungscharakteristik für beliebige transiente Signal. Dabei besteht die grundlegende Idee in einem auf der Korrelation basierenden Vergleich. Die Korrelation findet zwischen einem Referenzsignal und Signalen, welche innerhalb des nutzbaren Prüfvolumens der GTEM-Zelle gemessen werden, statt. Das Referenzsignal wird bei einer geringen Septumshöhe, in unmittelbarer Nähe des Speisepunktes, erfasst. Das gemessene Signal liegt im Vergleich zum eingespeisten Spannungssignal unverändert vor, da hier die Ausbreitung höherer Moden nicht möglich ist. Die Signale im Prüfvolumen werden entlang der in der IEC 61000-4-20 beschriebenen Gleichförmigkeitsebene erfasst. Der Korrelation geht eine Fensterung voraus, die die Signale auf ihre ursprüngliche Information reduziert und gleichzeitig eventuelle Einflüsse der GTEM-Zelle berücksichtigt. Unter Anwendung der Korrelation können anschließend Abweichungen zwischen den Signalen im Prüfvolumen und dem Referenzsignal erkannt werden.

Auf Basis dieser Methode ist die Übertragungscharakteristik der GTEM1250 für einen UWB-Puls und einen gedämpften Sinus ermittelt worden. Beide Signalformen werden mit einer hohen Formtreue übertragen. Dabei wird der UWB-Puls nahezu unverzerrt innerhalb des Zellenprüfvolumens abgebildet $(0,976 \leq \rho \leq 0,986)$, was sich mit der Validierung der Zelle nach Annex C deckt. Anhand der
Speisung des gedämpften Sinus wird diskutiert, inwiefern der UWB-Puls und dessen Darstellung im Prüfvolumen die Übertragung anderer transienter Signale mit einem Frequenzspektrum innerhalb des UBW-Puls Spektrums hinreichend beschreibt. Dafür werden anhand einer Auswertung der sekundären $E$-Feldkomponente $E_{z}$ im Frequenzbereich - gemäß den Vorgaben der IEC 61000-4-20 für die Validierung des TEM-Modes - eventuell kritische Frequenzen identifiziert. Zwar werden die Anforderungen im Frequenzbereich erfüllt, jedoch lässt sich bei einer Frequenz von $100 \mathrm{MHz}$ ein enormer Anstieg der $E_{z}$-Komponente feststellen. Die GTEM1250 überträgt einen DS mit einer Mittenfrequenz von $100 \mathrm{MHz}$ mit einer hohen Formtreue $(0,941 \leq \rho \leq 0,965)$, dennoch ist eine Abnahme des Korrelationskoeffizienten gegenüber der Übertragung des UWB-Pulses deutlich. Es lässt sich somit festhalten, dass der beschriebene UWB-Puls nicht sämtliche beliebige transiente Signal repräsentiert. Die hier vorgestellte Methode ermöglicht die Qualifizierung von TEMWellenleitern für jede beliebige transiente Signalform.

\section{Literatur}

1. IEC 61000-4-20 ed.2.0, Testing and measurement techniques Emission and immunity testing in transverse electromagnetric (TEM) waveguides. 2010.

2. C. A. Balanis. Antenna theory: analysis and design. WileyInterscience, 3 edition, 2005.

3. N. Briest, H. Garbe, and S. Potthast. Extension of the IEC 610004-20 Annex C to the Use of Arbitrary Transient Signals. In Electromagnetic Compatibility (EMC), 2016 IEEE International Symposium on, pages 829-834, 2016.

4. C. Kölling, D. Zamow, and H. Garbe. A correlation method to extend the IEC 61000-4-20 for UWB measurements. In EMC Europe 2011 York, pages 159-163, 2011.

5. M. Koch. Analytische Feldberechnung in TEM-Zellen. Shaker Verlag, 1998.

6. W. Radasky, K. Smith, D. Hansen, and D. Ristau. Calculations and measurements of fast EM pulses in the GTEM cell. In Electromagnetic Compatibility, 1996. Symposium Record. IEEE 1996 International Symposium on, pages 52-57, 1996. 


\section{Autoreninformationen}

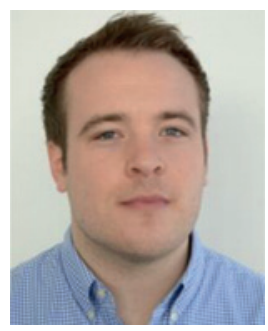

\section{Dipl.-Ing. Niklas Briest}

Institut für Grundlagen der Elektrotechnik und Messtechnik, Leibniz Universität Hannover briest@geml.uni-hannover.de

Niklas Briest wurde 1989 in Minden, Deutschland geboren. Er graduierte 2014 an der Leibniz Universität Hannover zum Dipl.-Ing. und ist seitdem am Institut für Grundlagen der Elektrotechnik und Messtechnik als Wissenschaftlicher Mitarbeiter im Fachgebiet der Elektromagnetischen Verträglichkeit angestellt.

Wissenschaftliche Schwerpunkte sind die Charakterisierung von TEM-Wellenleitern und Störfestigkeitsmessungen. Niklas Briest engagiert sich im Bereich der nationalen und internationalen Normung. National ist er Mitglied des Unterkomitees DKE 767.4, welches sich mit Messverfahren und Messgeräten zur Messung der elektromagnetischen Störemission oberhalb $9 \mathrm{kHz}$ beschäftigt. International ist er an der Entwicklung des TEMWellenleiter Standards IEC 61000-4-20 beteiligt und Mitglied der JWG TEM-Wellenleiter.

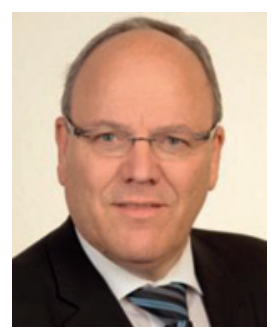

Prof. Dr.-Ing. Heyno Garbe Institut für Grundlagen der Elektrotechnik und Messtechnik, Leibniz Universität Hannover

Prof. Garbe wurde 1955 in Lauenau, Deutschland geboren. Während seiner Bundeswehrdienstzeit studierte er an der Universität der Bundeswehr in Hamburg Elektrotechnik. 1978 graduierter er dort zum Dipl.-Ing. und 1986 promovierte er zum Dr.-Ing. mit einer Arbeit aus dem Gebiet der Theoretischen Elektrotechnik.

1986 wechselte er ans BBC-Konzernforschungszentrum BadenDättwil (später ABB-Forschungszentrum). Dort war er in der Forschungsgruppe „Elektromagnetische Verträglichkeit“ von 1986 bis 1991 zuletzt als deren Leiter tätig. 1991 wurde die Hauptgruppe als eigenständige Firma EMC Baden AG mit ihm als Geschäftsführer ausgegliedert.
Seit 1992 ist er als Universitätsprofessor an Leibniz Universität Hannover, Institut für Grundlagen der Elektrotechnik, Fachgebiet (Lehrstuhl) „Elektromagnetische Verträglichkeit“ tätig.

Prof. Garbe engagiert sich weiterhin im Bereich der nationalen und internationalen Normung. National ist er Obmann des Unterkomitees DKE 767.4, welches sich mit Messverfahren und Messgeräten zur Messung der elektromagnetischen Störemission oberhalb $9 \mathrm{kHz}$ beschäftigt. International war und ist er maßgeblich an der Entwicklung des TEM-Wellenleiter Standards IEC 61000-4-20 beteiligt. 1988 wurde er Mitglied des IEEE. Dort hat er 2006 den höchsten Mitgliedsgrad Fellow erreicht. 2006 ist er auch als Ehrenmitglied der IEEE EMC Society ausgezeichnet worden. Aktuell ist er Vizepräsident der IEEE EMC Society.

Für seine Forschungsaktivitäten ist er von der IEEE EMC Society 2003 mit dem Laurence G. Cumming Award for Outstanding Service und 2010 mit dem Richard R. Stoddard Award for Outstanding Performance ausgezeichnet worden. Die Summation hat ihn 2002 zum EMP-Fellow ernannt.

Über 270 Beiträge in nationalen und internationalen peer-reviewten Publikationen verdeutlichen seine internationale Anerkennung.

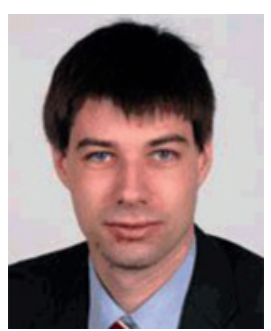

Dr.-Ing. Stefan Potthast

Wehrwissenschaftliches Institut für Schutztechnologien - ABC-Schutz, Munster

Dr. Potthast wurde in Paderborn, Deutschland geboren. Nach dem Studium der Physik an der Universität Paderborn erhielt er im Jahr 2003 den Abschluss als Diplom-Physiker und promovierte ebenfalls an der Universität Paderborn im Jahr 2007 zum Dr. rer. nat. am Department für Physik. In dieser Zeit umfasste seine Forschungsarbeit das epitaktische Wachstum kubischer GaN und AIGAN Heterostrukturen, so wie das Design und die Realisierung einer AIGaN/GaN basierten HEMT-Struktur.

In 2007 nahm er seine Tätigkeit beim damaligen Bundesamt für Wehrtechnik und Beschaffung (BWB, heute BAAINBw) auf. Seit 2008 ist er am Wehrwissenschaftlichen Institut für Schutztechnologien ABC-Schutz im Geschäftsfeld „Elektromagentische Effekte und HPEM“ beschäftigt. Schwerpunkte seiner Arbeit sind dort die Untersuchung der Wechselwirkung transienter, elektromagnetischer Felder mit elektronischen Komponenten und Systemen. 